\title{
Cdc42 is an antihypertrophic molecular switch in the mouse heart
}

\author{
Marjorie Maillet, ${ }^{1}$ Jeffrey M. Lynch, ${ }^{1}$ Bastiano Sanna, ${ }^{1}$ \\ Allen J. York, ${ }^{1}$ Yi Zheng, ${ }^{1}$ and Jeffery D. Molkentin',2 \\ 1Department of Pediatrics, University of Cincinnati, Division of Molecular Cardiovascular Biology, and Experimental Hematology and Cancer Biology, \\ Cincinnati Children's Hospital Medical Center, Cincinnati, Ohio, USA. ${ }^{2}$ Howard Hughes Medical Institute, Cincinnati, Ohio, USA.
}

\begin{abstract}
To improve contractile function, the myocardium undergoes hypertrophic growth without myocyte proliferation in response to both pathologic and physiologic stimulation. Various membrane-bound receptors and intermediate signal transduction pathways regulate the induction of cardiac hypertrophy, but the cardioprotective regulatory pathways or effectors that antagonize cardiac hypertrophy remain poorly understood. Here we identify the small GTPase $\mathrm{Cdc42}$ as a signaling intermediate that restrained the cardiac growth response to physiologic and pathologic stimuli. Cdc42 was specifically activated in the heart after pressure overload and in cultured cardiomyocytes by multiple agonists. Mice with a heart-specific deletion of $C d c 42$ developed greater cardiac hypertrophy at 2 and 8 weeks of stimulation and transitioned more quickly into heart failure than did wild-type controls. These mice also displayed greater cardiac hypertrophy in response to neuroendocrine agonist infusion for 2 weeks and, more remarkably, enhanced exercise-induced hypertrophy and sudden death. These pathologies were associated with an inability to activate JNK following stimulation through a MEKK1/ MKK4/MKK7 pathway, resulting in greater cardiac nuclear factor of activated T cells (NFAT) activity. Restoration of cardiac JNK signaling with an $M k k 7$ heart-specific transgene reversed the enhanced growth effect. These results identify what we believe to be a novel antihypertrophic and protective cardiac signaling pathway, whereby Cdc42-dependent JNK activation antagonizes calcineurin-NFAT activity to reduce hypertrophy and prevent transition to heart failure.
\end{abstract}

\section{Introduction}

The adult heart hypertrophies in response to environmental stress as a means of augmenting pump function and reducing wall stress (1). The heart undergoes pathologic cardiac hypertrophy in response to hypertension, genetic mutations in genes encoding sarcomeric proteins, or valvular disease or following myocardial infarction, all of which predispose to arrhythmia and sudden death and the development of heart failure $(2,3)$. The transition between pathological hypertrophy and heart failure is associated with a decrease of cardiac contractility, ventricular remodeling and fibrosis, as well as myocyte loss. The hypertrophic program is initiated at the cell membrane by receptors that bind various neurohormonal factors to activate specific intracellular signaling pathways. One of the most highly characterized hypertrophic signaling cascades involves the $\mathrm{Ca}^{2+}$-dependent serine/threonine protein phosphatase calcineurin (4). Elevated intracellular $\mathrm{Ca}^{2+}$ levels activate calcineurin, which then dephosphorylates the transcription factor nuclear factor of activated T cells (NFAT) in the cytoplasm, resulting in its translocation to the nucleus and the subsequent activation of hypertrophy-associated genes (5). MAPKs, such as ERK, JNK, and p38, are also activated by membrane-bound receptors on cardiomyocytes, where they then regulate aspects of the cardiac hypertrophic response $(6,7)$.

Surprisingly, the link between neuroendocrine factor-sensing receptors and calcineurin or MAPK signaling pathways is not well understood, although the small G proteins may be involved (8, 9). Cdc42 is a ubiquitously expressed member of the Rho family of small GTPases that, together with Rac1 and RhoA, have been

Conflict of interest: The authors have declared that no conflict of interest exists. Citation for this article: J. Clin. Invest. 119:3079-3088 (2009). doi:10.1172/JCI37694. implicated as signaling effectors in the heart $(8,10,11)$. Cdc42 cycles between a GDP-bound inactive state and a GTP-bound active state through the action of GTPase-activating proteins (GAPs), guanine nucleotide exchange factors (GEFs), and guanine nucleotide dissociation inhibitors (11-14). Cdc42 is dynamically regulated at the plasma membrane by GTP loading through cell type-specific GEFs and GAPs that respond to unique signals. Outside the heart, Cdc42 has multiple cellular functions, including the regulation of the actin and microtubule cytoskeleton formation that secondarily influences cellular morphology, polarity, migration, cell cycle progression, and cellular growth and death $(11,15)$. Analysis of gene-targeted mice has further verified many of these diverse functions for $C d c 42$, such as in maintaining the proper shape of keratinocytes and neuroepithelial cells as well as regulating hematopoietic stem cell maturation (16-20). By comparison, Cdc42GAP-deficient mice, which have enhanced Cdc42 activity, showed growth retardation, reduced cellular proliferation, and advanced aging $(21,22)$.

Very little is known about the function of Cdc42 in cardiomyocytes, although overexpression of $\mathrm{Cdc} 42$ was reported to enhance myocyte growth in culture, while overexpression of a dominantnegative Cdc42 mutant reduced leukemia inhibitory factorinduced (LIF-induced) myocyte growth (23). Here we investigate the role of $\mathrm{Cdc} 42$ in the adult mouse heart by using a conditional Cdc42-targeted allele in conjunction with a cardiac-specific cre transgenic line. In contrast to the single study in cultured myocytes discussed above (23), we observed that Cdc42 actually functions as an antihypertrophic molecular switch in the heart. Indeed, heartspecific deletion of $C d c 42$ enhances the cardiac growth response to both pathologic and physiological stimuli. Mechanistically, loss of Cdc42 renders JNK1/2 inactive to applied stimuli, and transgene- 


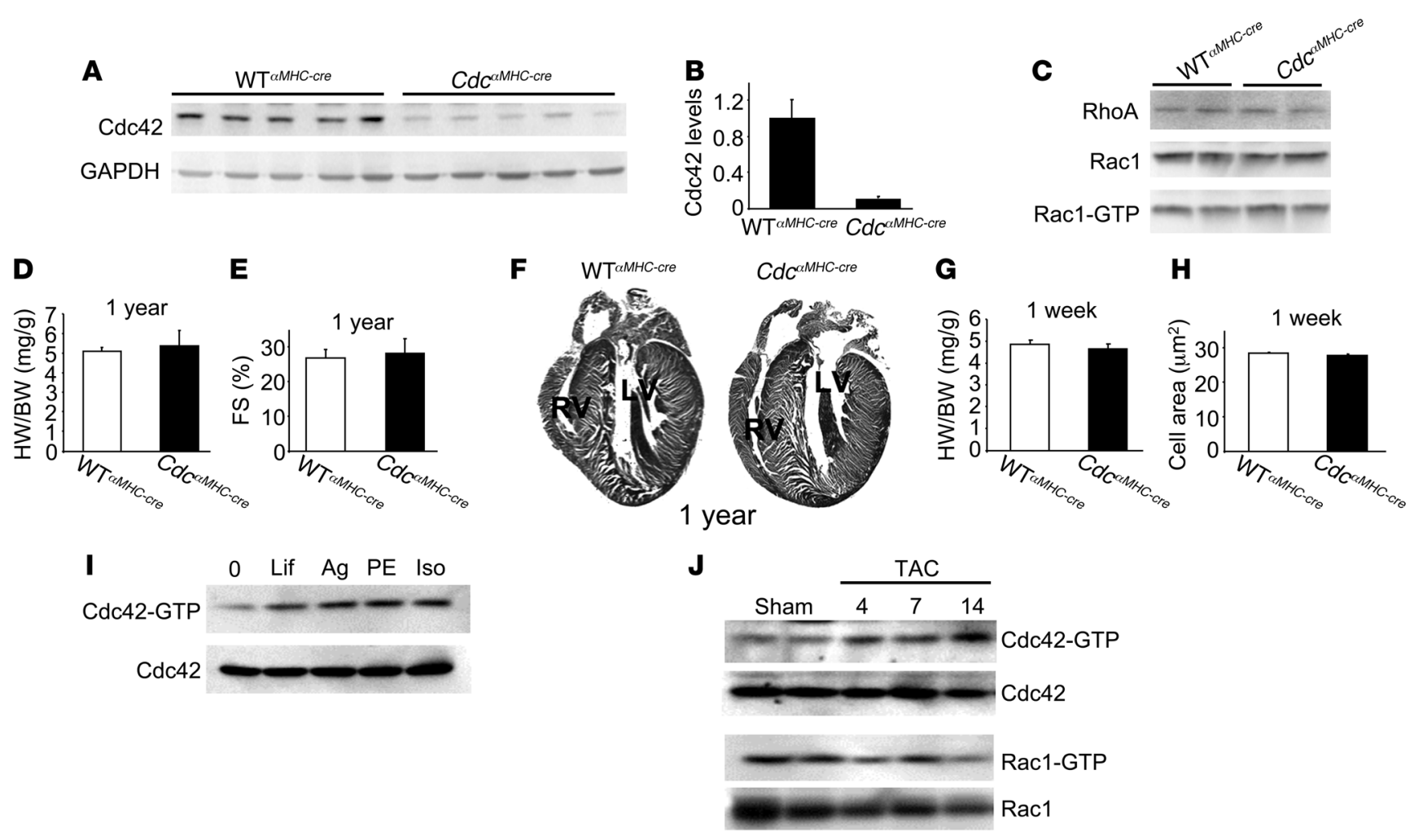

Figure 1

Generation of cardiac-specific Cdc ${ }^{\alpha M H C-c r e}$ mice. (A) Western blot analysis of Cdc42 protein levels in 2-month-old WT ${ }^{\alpha M H C-c r e}$ and Cdc ${ }^{\alpha M H C-c r e}$ hearts. GAPDH was used as a loading control. (B) Quantitation of the Western blot results shown in A. (C) Western blot analysis of total protein levels of RhoA and Rac1 in 2-month-old WT $\alpha M H C$-cre and Cdc ${ }^{\alpha M H C-c r e ~ h e a r t s . ~ R a c ~ b a s e l i n e ~ G T P ~ l o a d i n g ~ f o r ~ a c t i v i t y ~ w a s ~ a l s o ~ a s s a y e d . ~(D) ~ Q u a n t i f i c a t i o n ~ o f ~}$

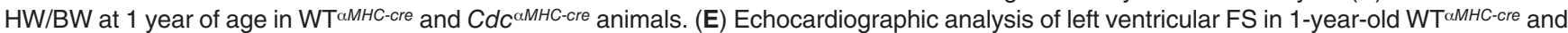
Cdc ${ }^{\alpha M H C-c r e}$ animals $\left(n=4\right.$ WT ${ }^{\alpha M H C-c r e}$ and $\left.n=3 C d c^{\alpha M H C-c r e}\right)$. (F) H\&E staining of longitudinal sections from hearts of WT ${ }^{\alpha M H C-c r e}$ and Cd $c^{\alpha M H C-c r e}$ mice at 1 year of age. (G) HW/BW at 1 week of age in WTaMHC-cre $(n=4)$ and CdcaMHC-cre $(n=6)$ mice. $(\mathbf{H})$ Myocyte cell surface areas in heart histological sections at 1 week of age in WT ${ }^{M M H C}$-cre and Cdc ${ }^{\alpha M H C-c r e}$ mice. (I) Cdc42-GTP pull-down activity assay in neonatal rat cardiomyocytes stimulated for 10 minutes with the indicated hypertrophic agonists at $1 \mu \mathrm{M}$. A representative Western blot of 3 independent experiments is shown. (J) Rac1-GTP and Cdc42-GTP pull-down activity assays from hearts of sham- or TAC-operated WT mice subjected to TAC for 4, 7, or 14 days.

mediated restoration in cardiac JNK activity reduced the enhanced growth effect associated with $\mathrm{Cdc} 42$ deletion. The exacerbated hypertrophic response associated with $C d c 42$ deletion increased NFAT transcriptional activity, and since JNKs normally antagonize NFAT activity, these results suggest what we believe is a novel antihypertrophic signaling network in the heart.

\section{Results}

Generation and characterization of $C d c^{\alpha M H C-c r e}$ mice. Conventional gene targeting of $C d c 42$ results in early embryonic lethality, precluding an analysis of $\mathrm{Cdc} 42$ function in adult disease-related processes (24). To circumvent this limitation, the $C d c 42$ gene was targeted with LoxP sites to permit tissue-specific deletion in a conditional manner through cre-mediated excision $(17,19,20)$. We investigated the function of $\mathrm{Cdc} 42$ in the adult mouse heart by crossing the Cdc42-LoxPtargeted allele with transgenic mice expressing cre under the control of the cardiac-specific $\alpha$-myosin heavy chain $(\alpha-\mathrm{MHC})$ promoter (Cdc $c^{\alpha M H C-c r e}$ mice). $C d c^{\alpha M H C-c r e}$ mice were born at the expected Mendelian ratios and were overtly normal. Hearts from 2-month-old $C d c^{\alpha M H C-c r e}$ mice showed an $88 \%$ deletion of Cdc42 protein compared with WT ${ }^{\alpha M H C-c r e}$ mice (Figure 1, A and B). Earlier and more efficient deletion of $C d c 42$ using the Nkx2.5-cre knockin allele resulted in early embryonic lethality, indicating that complete loss of Cdc42 from the heart at this early time point was not tolerated, necessitating our use of the $\alpha$-MHC-cre transgene (data not shown). Deletion of $C d c 42$ in myocytes of the adult heart did not influence protein expression of the related small GTPase family members RhoA or Rac1 or the activated form of Rac1 bound to GTP (Figure 1C). Cardiac phenotyping in $C d c^{\alpha M H C-c r e}$ mice at 1 year of age revealed no difference in heart weight normalized to body weight (HW/BW), ventricular fractional shortening (FS) measured by echocardiography, or ventricular and cellular morphologies measured by histological methods (Figure 1, $\mathrm{D}-\mathrm{F}$, and Table 1). We also investigated whether deletion of $\mathrm{Cdc} 42$ from the heart might alter myocyte number. Hearts were harvested from 1-week-old Cdc $c^{\alpha M H C-c r e}$ and WT ${ }^{\alpha M H C-c r e}$ mice, and heart weights were measured and myocyte surface areas quantified from histological sections (Figure 1, G and $\mathrm{H}$ ). No differences were noted in heart weights or cellular surface areas of $C d c^{\alpha M H C-c r e}$ mice compared with controls at 1 week of age, suggesting that total myocyte content was unchanged. Thus, deletion $\mathrm{Cdc} 42$ with the $\alpha-\mathrm{MHC}$-cre transgene did not appreciably alter the baseline phenotype of the heart.

We were also interested in determining whether endogenous Cdc42 might be activated in response to hypertrophic stimulation to the heart or myocytes in culture. Indeed, stimulation of neonatal 


\section{Table 1}

Echocardiographic assessment of cardiac structure and function in 1-year-old WT $\alpha$ MHC-cre and CdcaMHC-cre mice

\begin{tabular}{lccccc}
\hline Mouse & IVSth $(\mathbf{m m})$ & LVPWth $(\mathbf{m m})$ & LVED $(\mathbf{m m})$ & LVES $(\mathbf{m m})$ & FS $(\%)$ \\
WTaMHC-cre $(n=4)$ & $0.69 \pm 0.05$ & $0.61 \pm 0.03$ & $4.63 \pm 0.27$ & $3.32 \pm 0.28$ & $28.67 \pm 2.04$ \\
Cdc aMHC-cre $^{\prime}(n=3)$ & $0.73 \pm 0.03$ & $0.70 \pm 0.03^{A}$ & $4.53 \pm 0.36$ & $3.28 \pm 0.46$ & $28.24 \pm 4.29$ \\
\hline
\end{tabular}

AP $<0.05$. All measurements are means \pm SEM, and significance was determined using the Student's $t$ test. Septal and left ventricular wall thicknesses were assessed in diastole. IVSth, intraventricular septal thickness; LVPWth, left ventricular posterior wall thickness; LVED, left ventricular end-diastolic dimension; LVES, left ventricular end-systolic dimension.

rat cardiomyocytes in culture for 10 minutes with LIF, angiotensin II (Ag), phenylephrine (PE), or isoproterenol (Iso) each increased the GTP-bound, activated state of Cdc42 (Figure 1I). Cdc42 activation (GTP bound) was also increased in hearts of WT mice subjected to transverse aortic constriction (TAC) for 4, 7, and 14 days compared with hearts from sham-operated mice (Figure 1J). By comparison, Rac1 activation was not increased and in fact appeared to be decreased in the hearts of mice subjected to TAC (Figure 1J).

Deletion of Cdc42 from the adult heart exacerbates pressure overload bypertrophy. Given the results presented above, we hypothesized that Cdc42 might be more specialized as a signaling adaptor that connects neuroendocrine and stress-related membrane receptor activation to downstream pathways that program hypertrophy and/or dilated cardiomyopathy. Pressure overload by TAC was therefore performed in 2-month-old WT ${ }^{\alpha M H C-c r e}$ and $C d c^{\alpha M H C-c r e}$ mice. Mice were sacrificed 2 weeks later and showed significant increases in the HW/BW ratio in both groups compared with sham-operated controls (Figure 2A). Interestingly, the amount of cardiac hypertrophy in $C d c^{\alpha M H C-c r e}$ mice was significantly greater than in WT $\mathrm{WTHC}^{\alpha M r e}$ mice (Figure 2A). This greater hypertrophy response was not due to a difference in the TAC procedure, as the TAC pressure gradients were similar between the 2 groups (Table 2). Analysis of myocyte areas from histological sections after 2 weeks of TAC also revealed greater cellular hypertrophy in $C d c^{\alpha M H C-c r e}$ myocytes compared with WT ${ }^{\alpha M H C-c r e}$ (Figure 2B). The exacerbated hypertrophy observed in $C d c^{\alpha M H C-c r e}$ hearts was not initially associated with a reduction in FS after 2 weeks of TAC (Table 2). However, histological analysis of fibrosis by Masson's trichrome staining showed a 6-fold increase in collagen deposition in the hearts of CdcaMHC-cre mice after TAC compared with WT ${ }^{\alpha M H C-c r e}$ (Figure 2, C and D). Deletion of Cdc42 with the $\alpha$-MHC-cre transgene also enhanced levels of apoptosis and necrosis after TAC stimulation compared with controls. Apoptosis was directly measured by TUNEL from histological sections (Figure $2 \mathrm{E}$ ), while necrosis was indirectly evaluated by assessing Evan's blue dye (EBD) uptake in vivo, followed by histological analysis (Figure 2F). A mouse model of muscular dystrophy with known membrane leakiness, $m d x$, was used as a control for EBD uptake. Both $C d c^{\alpha M H C-c r e}$ and $m d x$ mice showed baseline EBD uptake that was significantly enhanced by TAC (Figure 2F). Thus, deletion of Cdc42 from the heart promoted greater cardiac hypertrophy following 2 weeks of TAC stimulation, and while function was not compromised at this time point, other indices of disease were enhanced.

Cardiac-specific Cdc ${ }^{\alpha M H C-c r e}$ mice are more susceptible to beart failure. To more carefully extend the results presented above after 2 weeks of TAC, we subjected 2-month-old WT $\mathrm{T}^{\alpha M H C-c r e}$ and $C d c^{\alpha M H C-c r e}$ mice to long-term (8-week) pressure overload stimulation. Eight weeks of TAC also produced significantly greater increases in HW/BW ratios and myocyte cross-sectional areas in $C d c^{\alpha M H C-c r e}$ mice compared with WT $\mathrm{WMHC}^{\mathrm{M} r e}$ controls (Figure 3, A and B). After 8 weeks of TAC, WT ${ }^{\alpha M H C-c r e}$ mice showed a significant reduction in FS, yet this decrease was significantly greater in $C d c^{\alpha M H C-c r e}$ mice, suggesting that deletion of $\mathrm{Cdc} 42$ in the heart predisposed to failure (Figure $3 \mathrm{C}$ ). Measurement of TAC pressure gradients was performed after 2 weeks of TAC in these mice, revealing no differences (data not shown). Lung weight normalized to body weight (LW/BW) was significantly elevated in $C d c^{\alpha M H C-c r e}$ mice after 8 weeks of TAC, indicating pulmonary congestion due to poor cardiac function (Figure 3D). Consistent with this increased cardiac disease profile, cardiac fibrosis was greater in $C d c^{\alpha M H C-c r e}$ mice compared with WT ${ }^{\alpha M H C-c r e}$ mice after 8 weeks of TAC (Figure 3E). Analysis of hypertrophic/ stress responsive gene expression in the heart by RT-PCR analysis also showed a greater response in CdcaMHC-cre mice after 8 weeks of TAC compared with WT ${ }^{\alpha M H C-c r e}$ mice (Figure 3F). Collectively, these results indicate that deletion of $\mathrm{Cdc} 42$ from the heart with the $\alpha$-MHC-cre transgene predisposed to a greater hypertrophic response that transitioned more readily to heart failure, suggesting that $\mathrm{Cdc} 42$ is normally protective and antihypertrophic.

Cardiac-specific Cdc ${ }^{\alpha M H C-c r e}$ mice have exacerbated pathologic and physiologic hypertrophy. In addition to pressure overload stimulation, we also investigated the response of $C d c^{\alpha M H C-c r e}$ mice to neuroendocrine infusion and physiologic hypertrophy stimulation by exercise. First, chronic Ag and PE co-infusion was performed for 2 weeks with Alzet minipumps, revealing a significantly greater hypertrophic response in $C d c^{\alpha M H C-c r e}$ mice compared with WT ${ }^{\alpha M H C-c r e}$ control mice (Figure 4, A and B). $C d c^{\alpha M H C-c r e}$ and control mice were also subjected to forced swimming exercise daily for 3 weeks. Remarkably, two-thirds of the $C d c^{\alpha M H C-c r e}$ mice died suddenly during the swimming protocol, compared with no death in WTaMHC-cre controls or rested mice (Figure 4C). While we are uncertain exactly why $C d c^{\alpha M H C-c r e}$ mice perished during exercise, the remaining mice in this cohort showed significantly greater increases in HW/BW ratios and myocyte surface areas from histological sections compared with the WT ${ }^{\alpha M H C-c r e}$ control group (Figure 4, D and E). Despite this greater increase in hypertrophy following swimming, cardiac FS was not reduced in $C d c^{\alpha M H C-c r e}$ mice, nor was there any evidence of histopathology such as fibrosis or TUNEL (Figure 4, F-H). Taken together, these results further suggest that Cdc42 is an antihypertrophic mediator that also functions during physiologic exercise stimulation to partially limit heart growth.

Deletion of $\mathrm{Cdc} 42$ from the heart alters JNK signaling. To investigate the potential mechanism whereby loss of $C d c 42$ promoted greater hypertrophy and predisposition to heart failure, we surveyed downstream signaling pathways. Most notably, Cdc42 was previously shown to affect MAPK signaling pathways, which are known to affect cellular growth $(11,25)$. Here we observed a prominent defect in JNK phosphorylation in the hearts of Cdc ${ }^{\alpha M H C-c r e}$ mice after 2 and 7 days of TAC stimulation compared with WT ${ }^{\alpha M H C-c r e}$ controls, while phosphorylation of p38, ERK1/2, Akt, and glyco- 

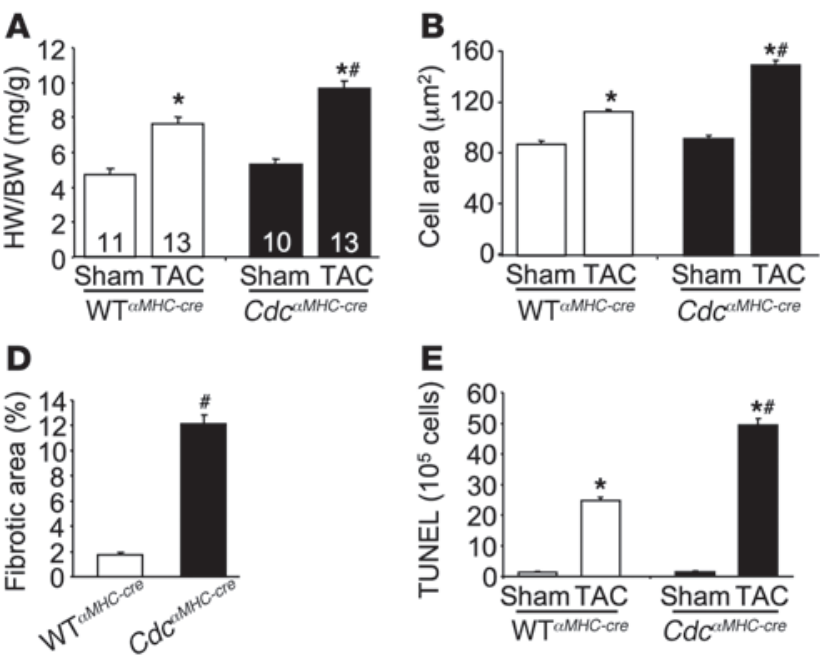
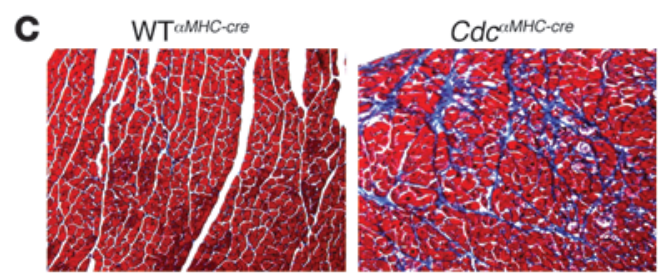

$\mathbf{F}$

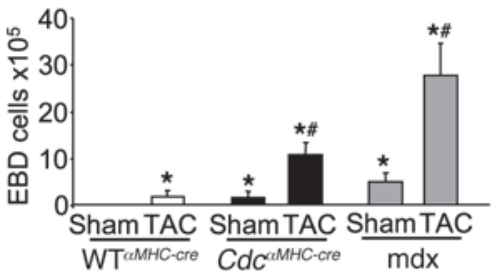

\section{Figure 2}

Exacerbated hypertrophy in Cdc $c^{\alpha M H C-c r e}$ hearts. (A) Quantification of HW/BW in 2-month-old WT $\alpha M H C$-cre and Cdc $\alpha M H C$-cre mice subjected to TAC for 2 weeks or a sham surgical procedure. The number of mice analyzed in each group is shown in each bar. ${ }^{\star} P<0.05$ versus sham; ${ }^{*} P<0.05$ versus TAC WT ${ }^{\alpha M H C}$-cre. (B) Quantification of myocyte surface area from cardiac histological sections of sham- or TAC-operated WT ${ }^{\alpha M H C-c r e}$ and Cdc ${ }^{\alpha M H C-c r e}$ mice ( $n \geq 500$ cells from at least 3 different animals per group). ${ }^{*} P<0.05$ versus sham; $\#<0.05$ versus TAC WT $\alpha M H C-c r e$. (C) Histological view of cardiac fibrosis stained in blue by Masson's trichrome in WT ${ }^{\alpha M H C-c r e}$ and Cdc ${ }^{\alpha M H C-c r e}$ mice subjected to TAC for 2 weeks (original magnification, $\times 200)$. (D) Quantitation of fibrosis induced by 2 weeks of TAC in hearts of WT $\alpha M H C$-cre and Cdc $\alpha M H C$-cre mice $(n=3)$. "\# $P<0.05$ versus WTaMHC-cre. (E) Immunohistochemical assessment of TUNEL in the hearts of WTaMHC-cre and CdcaMHC-cre mice subjected to 1 week of TAC (at least 100,000 nuclei were counted; $n=3$ mice). (F) Quantification of EBD uptake in WT $\alpha M H C$-cre and CdcaMHC-cre hearts subjected to TAC for 1 week or a sham procedure. Dystrophin-deficient $m d x$ mice were used as a positive control (at least 100,000 cells from 3 different mice were analyzed). ${ }^{*} P<0.05$ versus sham; ${ }^{\#} P<0.05$ versus TAC WT ${ }^{\alpha M H C}$-cre.

gen synthase kinase $3 \alpha / \beta($ GSK3 $\alpha / \beta)$ were unaltered (Figure $5 \mathrm{~A})$. We repeated the assay for phosphorylated JNK a second time from different extracts and again observed a defect in activation after TAC in CdcaMHC-cre mice (Figure 5A). Moreover, direct examination of JNK activity by measuring phosphorylation of GST-cJun in vitro showed a significant reduction in the hearts from $C d c^{\alpha M H C-c r e}$ mice after TAC (Figure 5B). We also instituted an in vitro approach in cultured neonatal rat cardiomyocytes using a lentivirus-encoding shRNA construct against Cdc42 to reduce the expression/activity of this protein. The shRNA reduced Cdc42 protein levels by approximately $74 \%$, which correlated with a reduction in JNK phosphorylation after LIF and Iso stimulation (Figure 5C). We used these samples to examine the activity of upstream signaling components that might directly respond to Cdc42. Interestingly, the direct Cdc42 target, MEKK1, also showed reduced phosphorylation after agonist stimulation in the presence of the Cdc42 shRNA (Figure 5C). Downstream of MEKK1 but directly upstream of JNK1/2, MKK7 phosphorylation was similarly reduced with Cdc42 shRNA lentiviral infection after agonist stimulation (Figure 5C). We also observed a similar reduction in MKK4 activation after LIF stimulation (data not shown). Taken together, these data suggest that Cdc42 signals directly to MEKK1 in cardiomyocytes, which in turn alters MKK4/7 activity, leading to the observed alteration in JNK activity. Indeed, Sadoshima and colleagues showed that $M E K K 1^{-/-}$mice had significantly reduced JNK activation in the heart after TAC stimulation (26). Finally, no changes in p21-activated kinases (PAK) or focal adhesion kinase (FAK) were observed in this assay (Figure 5C).

$J N K$ is a critical effector of $C d c 42$ in regulating cardiac bypertrophy. JNK was selected for more thorough investigation because we previously showed that inhibition of this kinase promoted greater car- diac hypertrophy in both dominant-negative transgenic mice and Jnk1/2 gene-targeted mice (27). We used $\alpha$-MHC-MKK7 transgenic mice, which are known to have a 4-fold increase in JNK activity (28), to enhance JNK signaling in the $C d c^{\alpha M H C-c r e}$ background. At baseline, MKK7 transgenic mice did not present with appreciable cardiac hypertrophy when crossed into the Cdc42-loxP-targeted background, either with or without the $\alpha-\mathrm{MHC}$-cre transgene (Figure 6 , A and C). However, the enhanced growth response observed in $C d c^{\alpha M H C-c r e}$ mice after 2 weeks of TAC was reduced to WT ${ }^{\alpha M H C-c r e}$ TAC levels with the MKK7 transgene (Figure 6, A and C). All cohorts subjected to TAC showed similar TAC pressure gradients, indicating that the observed differences in organ and cellular hypertrophy was not caused by disparity in the pressure overload stimulation (Figure 6B). Similarly, the enhancement in cardiac fibrosis in $C d c^{\alpha M H C-c r e}$ mice after TAC was reduced to baseline levels by the MKK7 transgene (Figure 6E). Taken together, these results suggest that reestablishing JNK signaling downstream of Cdc42 deletion normalizes the cardiac hypertrophic response in $C d c^{\alpha M H C-c r e}$ hearts.

One potential mechanism whereby decreased JNK activity might promote greater cardiac hypertrophy is by permitting greater NFAT activity following stress stimulation. Indeed, JNK is known to directly phosphorylate NFAT transcription factors to antagonize their nuclear translocation and induction of hypertrophic genes $(27,29,30)$. We previously showed that JNK-inhibited mice had greater NFAT activity in the heart (27). Here we introduced the NFAT-luciferase reporter transgene in the WT $\alpha M H C$-cre and $C d c^{\alpha M H C-c r e}$ backgrounds. As predicted, NFAT-luciferase activity was significantly higher in $C d c^{\alpha M H C-c r e}$ hearts compared with WT ${ }^{\alpha M H C-c r e}$ after 2 weeks of TAC stimulation (Figure 6D). Importantly, the NFAT-luciferase transgene was neutral and did not influence the hypertrophic response of WT ${ }^{\alpha M H C-c r e}$ and $C d c^{\alpha M H C-c r e}$ mice following 
Table 2

Echocardiographic assessment of cardiac structure and function before and after 2 weeks of TAC

\begin{tabular}{|c|c|c|c|c|c|c|}
\hline Mouse & IVSth (mm) & LVPWth (mm) & LVED (mm) & LVES (mm) & FS (\%) & $P G(m m H g)$ \\
\hline WTaMHC-cre sham $(n=11)$ & $0.73 \pm 0.04$ & $0.78 \pm 0.04$ & $3.77 \pm 0.1$ & $2.49 \pm 0.09$ & $34 \pm 1.7$ & \\
\hline WT $\alpha M H C-c r e \operatorname{TAC}(n=13)$ & $0.75 \pm 0.03$ & $0.83 \pm 0.05$ & $3.69 \pm 0.08$ & $2.51 \pm 0.07$ & $31.98 \pm 0.9$ & $66.2 \pm 4$ \\
\hline CdcaMHC-cre sham $(n=10)$ & $0.7 \pm 0.03$ & $0.86 \pm 0.05$ & $3.74 \pm 0.13$ & $2.43 \pm 0.13$ & $35.33 \pm 1.6$ & \\
\hline Cdc $c^{\alpha M H C-c r e} \operatorname{TAC}(n=13)$ & $1.03 \pm 0.06^{A, B}$ & $1 \pm 0.05^{A, B}$ & $3.6 \pm 0.08$ & $2.5 \pm 0.08$ & $30.78 \pm 1.1$ & $72 \pm 4.6$ \\
\hline
\end{tabular}

${ }^{A} P<0.05$ versus sham. ${ }^{B} P<0.05$ versus $W^{\alpha M H C}$-cre TAC. All measurements are means \pm SEM, and significance was assayed by 1 -way ANOVA. Septal and left ventricular wall thicknesses were assessed in diastole. PG, pressure gradient.

2 weeks of TAC, except that $C d c^{\alpha M H C-c r e}$ mice continued to show greater hypertrophy (Figure 6F). In conclusion, targeted deletion of the small GTPase $C d c 42$ from the adult myocardium allows greater cardiac hypertrophy following stress stimulation, in part through defective JNK signaling, which renders NFAT more active.

\section{Discussion}

Here we show that the loss of $C d c 42$ renders the mouse heart more capable of hypertrophic growth following pressure overload, neuroendocrine agonist infusion, and exercise, suggesting that it normally functions to antagonize cardiac growth when activated. This proposed function for $\mathrm{Cdc} 42$ is unique among the Rho GTPases, as Ras, Rac1, and RhoA have all been shown to facilitate cardiac hypertrophy or myopathy in genetically modified mice. For example, overexpression of activated Ras in the hearts of transgenic mice generated cardiac hypertrophy that was pathologic and associated with reduced sarcoplasmic reticulum calcium handling (31). Similarly, Rac1 overexpression in transgenic mice induced early cardiac hypertrophy or dilated heart failure (32). In support of these results, in one study, cardiac-specific deletion of Rac1 in mice resulted in reduced cardiac growth at baseline associated with reduced NADPH oxidase activity (33). In contrast, another study found that RhoA overexpression in the hearts of transgenic mice did not produce left ventricular hypertrophy, although it did mediate atrial enlargement, alterations in the cardiac conduction system, and ventricular dilation with aging (34). By comparison, in the present study, Cdc42 appeared to be functionally distinct from the other Rho GTPases, where it served as an antihypertrophic and protective mediator during stress stimulation, and it had no reported linkage to NADPH oxidase activity. However, the observed protective and antihypertrophic function for $\mathrm{Cdc} 42$ in the heart is inconsistent with studies conducted in cultured cardiomyocytes with adenoviral gene transfer of dominant-negative and consti-

\section{Figure 3}

Cdc ${ }^{\alpha M H C-c r e}$ mice are more susceptible than WT ${ }^{\alpha M H C-c r e}$ mice to heart failure. (A) Quantification of HW/BW in 2-month-old WTaMHC-cre and Cdc ${ }^{\alpha M H C-c r e}$ mice subjected to TAC for 8 weeks compared with sham operation. The number of mice analyzed in each group is shown inside the bars. (B) Myocyte surface areas from histological sections of mice described in $\mathbf{A}$ ( $n \geq 500$ cells from at least 3 mice per group). (C) Echocardiographic assessment of FS after a sham procedure or 8 weeks of TAC for the mice described in A and B. (D) Quantification of LW/BW after 8 weeks of TAC for the mice described in A and B. (E) Quantitation of fibrosis induced by 8 weeks of TAC in hearts of WTaMHC-cre and Cdc c ${ }^{\alpha M H C \text {-cre }}$ mice $(n=3) .{ }^{\star} P<0.05$ versus sham; $\# P<0.05$ versus TAC WT $\alpha M H C$-cre. (F) RT-PCR of mRNA from the hearts of mice described in A for ANP, BNP, $\beta-\mathrm{MHC}, \alpha-S k a$, and the L7 control RNA. tutively active Cdc42 mutants. Those studies suggested that Cdc42 regulates myocyte growth by the addition of sarcomeres in series, although the disparity with our study may simply reflect the different experimental systems employed (23).

While a large number of pro-hypertrophic signaling pathways have been proposed and validated in genetically modified mouse models, surprisingly few antihypertrophic signaling pathways have been described. Perhaps the only well-described antihypertrophic regulatory pathway to date is that involving cGMP. Increased levels of cGMP in cardiomyocytes through stimulation of the atrial natriuretic peptide (ANP) receptor, enhanced activity of cytosolic guanylyl cyclases through nitric oxide, or inhibition of select phosphodiesterases (PDEs) renders myocytes less able to hypertrophy to stress or neuroendocrine agonist stimulation. Increased intracellular cGMP results in cGMP-dependent protein kinase type I (PKG) activation and inhibition of cardiac hypertrophy, in part, by inhibition of calcineurin-NFAT signaling (35). Indeed, inhibition of PDE5A with sildenafil, which increases total cGMP and leads to PKG activation, renders the heart protected from pressure overload stimulation and subsequent hypertrophy (36).
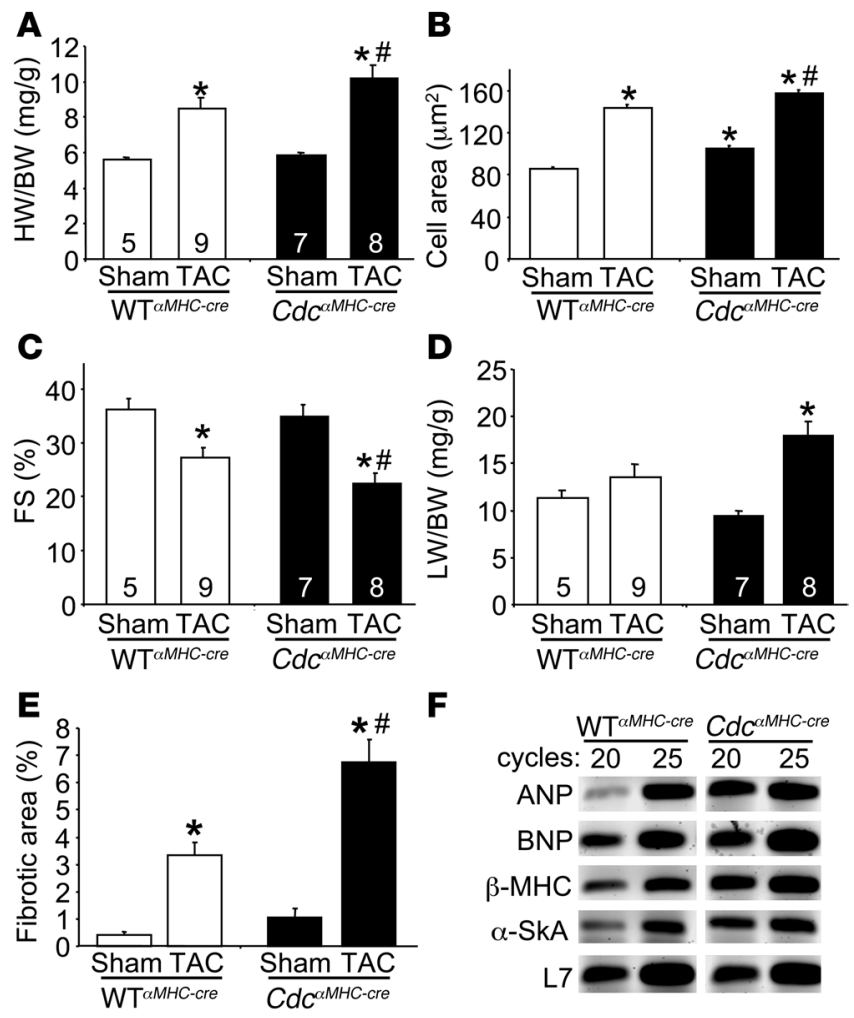

F

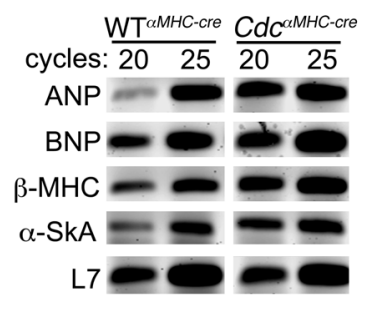


A
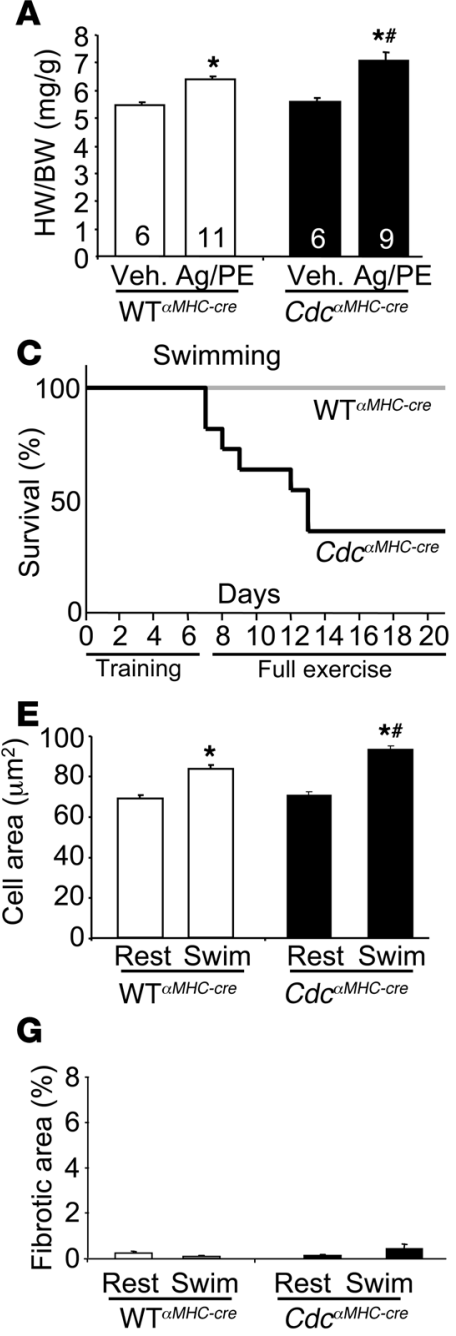

B

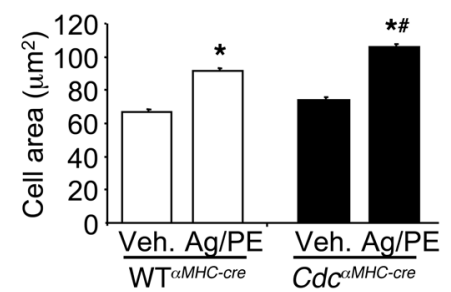

D

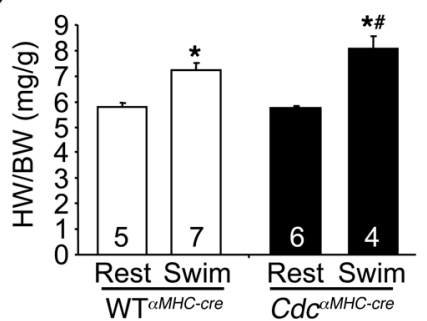

$\mathbf{F}$

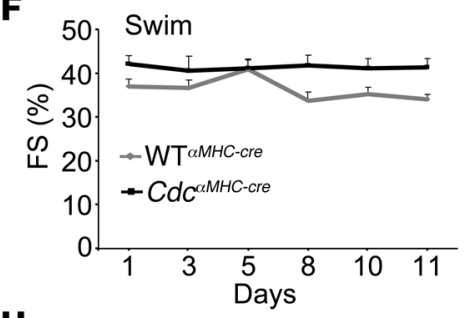

H

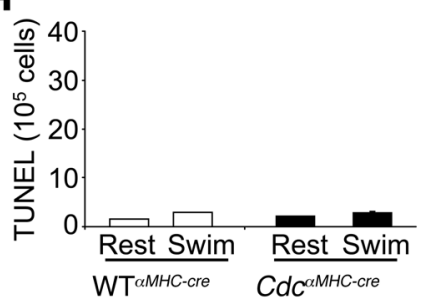

Figure 4

Cdc ${ }^{\alpha M H C-c r e}$ mice show greater hypertrophy to agonist and exercise stimulation. (A) Quantitation of HW/BW in 2-monthold WTaMHC-cre and CdcaMHC-cre mice stimulated for 2 weeks with $\mathrm{Ag}(432 \mu \mathrm{g} / \mathrm{kg} / \mathrm{d})$ and PE $(100 \mathrm{mg} / \mathrm{kg} / \mathrm{d})$ or vehicle (Veh) released by Alzet osmotic mini-pumps. The number of mice analyzed in each group is shown inside each bar. ${ }^{*} P<0.05$ versus vehicle; ${ }^{\#} P<0.05$ versus $\mathrm{Ag} / \mathrm{PE} W \mathrm{WT}^{\alpha M H C-c r e}$. (B) Quantitation of myocyte surface areas from the mice shown in A. At least 500 myocytes were quantified from 3 different mice in each group. ${ }^{\star} P<0.05$ versus vehicle; $\# P<0.05$ versus Ag/PE WT $\alpha M H C$-cre. (C) Survival in 2-month-old WT $\alpha M H C$-cre and $\mathrm{Cdc} \mathrm{c}^{\alpha \mathrm{MHC}-\mathrm{cre}}$ subjected to 3 weeks of daily swimming (initially, $n=7$ WT ${ }^{\alpha M H C-c r e}, n=12$ Cdc $\left.{ }^{\alpha M H C-c r e} ; P<0.001\right)$. (D) Quantitation of HW/BW in resting WT ${ }^{\mathrm{MHHC}-\mathrm{cre}}$ and $\mathrm{Cd} \mathrm{C}^{\alpha M H C-c r e}$ mice and in the remaining exercised animals. ${ }^{\star} P<0.05$ versus rest; $\# P<0.05$ versus swim WTaMHC-cre. (E) Quantitation of myocyte surface areas from the mice described in D. ${ }^{*} P<0.05$ versus rest; ${ }^{*} P<0.05$ versus swim WT ${ }^{4 M H C-c r e}$. (F) Echocardiographic assessment of FS in WTaMHC-cre and Cdc ${ }^{\alpha M H C-c r e}$ mice over 11 days of swimming. ( $\mathbf{G}$ and $\mathbf{H}$ ) Analysis of cardiac fibrosis and TUNEL after swimming in heart histological sections from mice in the indicated groups.
In addition to cGMP/PKG signaling, terminal activation of p38 or JNK may have an antihypertrophic effect on the heart under select conditions. For example, expression of dominant-negative p38 or JNK in the hearts of transgenic mice, or even targeted deletion of $J n k 1 / 2$ or $p 38 \alpha$ permitted greater cardiac hypertrophy following pressure overload stimulation, exercise, or with neuroendocrine-like agonists $(27,37,38)$. This increase in cardiac hypertrophy associated with $\mathrm{p} 38$ or JNK inhibition was reversed by deletion of calcineurin $A \beta$, showing that calcineurin-NFAT signaling was a mechanism whereby these kinases mediated their antihypertrophic effect. That JNK and p38 can have an antihypertrophic effect is also somewhat consistent with the phenotype of mice with MKK3/6 or MKK7 overexpression in the heart, which showed severe cardiac dilation and failure without prominent hypertrophy $(39,40)$. We also previously showed that Jnk1 $1^{+-} J n k 2^{-/-}$mice developed spontaneous cardiac hypertrophy with aging and that young mice subjected to TAC had a greater hypertrophic response (27). The latter result is especially important, as it is obviously specific to JNK signaling and is not confounded by divergent signaling that characterizes other effectors used to examine this pathway. For example, overexpression of dominant-negative MKK4 diminished cardiac hypertrophy in a rat model of pressure overload, although this effector is not completely JNK specific (41). Our results are consistent with data from $M E K K 1^{-/-}$mice, which, like $C d c^{\alpha M H C-c r e}$ mice, showed less JNK activation after TAC stimulation and greater cardiac hypertrophy at the 2 -week time point (26). However, Schneider and colleagues showed that hypertrophy and heart disease observed in Gaq-overexpressing transgenic mice was rescued by crossing into the $M E K K 1^{-/-}$background (42). These seemingly discordant results are obviously confusing, but perhaps JNK signaling plays both roles (pro- and antihypertrophic), depending on the activity of parallel regulatory pathways, the duration and/or strength of JNK signaling, or the intracellular location of activated JNK associated with different upstream effector pathways. It is also formally possible that other signaling effectors are more critical in regulating cardiac hypertrophy downstream of Cdc42.

Our results suggest a straightforward pathway in which Cdc42 receives signals from membrane-bound receptors to initiate the JNK cascade through MEKK1. Indeed, MEKK1 is known to associate with Cdc42 and to be directly activated by it (43), and others have shown a prominent link between Cdc42 and JNK activity (44, 45). Once activated by Cdc42, MEKK1 can directly phosphorylate and activate MKK4 and MKK7, which in turn directly phosphorylate JNK1/2. Previous work in mouse embryonic fibroblasts showed that loss of $\mathrm{Cdc} 42$ attenuates JNK phosphorylation, whereas activation of $\mathrm{Cdc} 42$ in $\mathrm{Cdc} 42 \mathrm{GAP} \mathrm{P}^{-/-}$mouse embryonic 
A

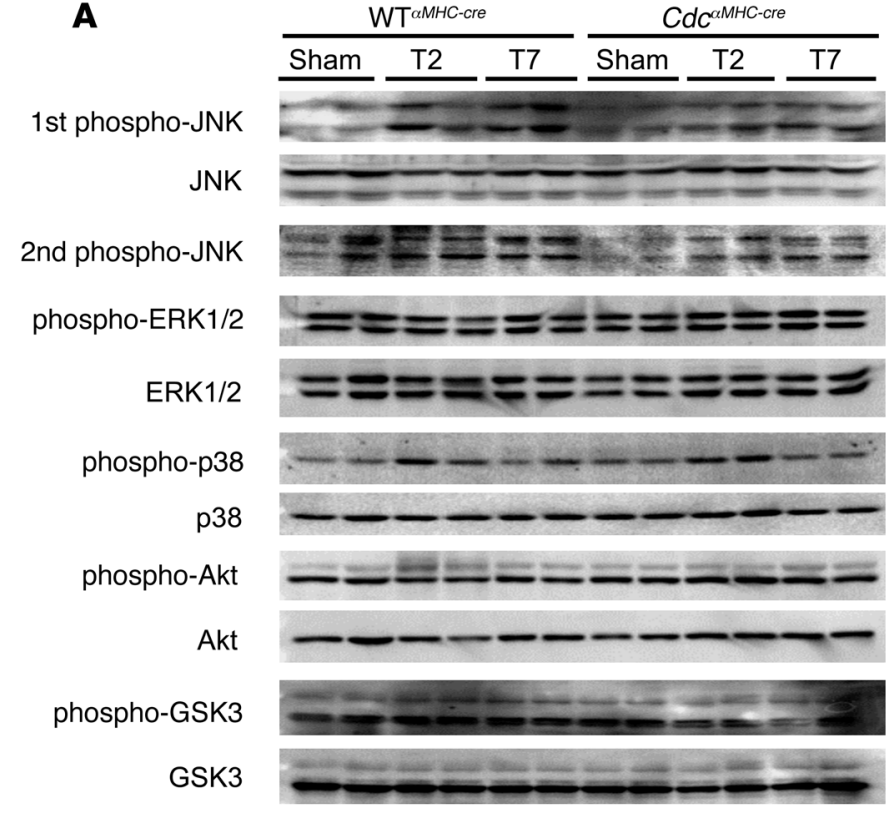

B

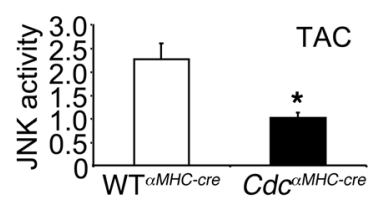

\section{Figure 5}

Inhibited JNK activation following TAC in CdcaMHC-cre mice. (A) Western blotting from heart protein extracts for phosphorylated and total JNK, p38, ERK1/2, Akt, and GSK3 in 2-month-old WT $\alpha M H C$-cre and Cdc ${ }^{\alpha M H C-c r e ~ m i c e ~ s u b j e c t e d ~ t o ~ T A C ~ f o r ~} 2$ days (T2) or 7 days (T7) versus sham-operated mice. A representative Western blot of 3 independent experiments is shown. A second Western blot for phospho-JNK1/2 was generated from different mice. (B) Quantitation of JNK1/2 activity determined by radioactive detection of GST-cJun phosphorylation from protein extracts after 1 week of TAC in WT ${ }^{\alpha M H C}$-cre or $C d c^{\alpha M H C-c r e}$ hearts. ${ }^{*} P<0.05$ versus WT ${ }^{\alpha M H C}$-cre. . (C) Western analysis of total Cdc42 protein levels and various kinases in protein extracts generated from neonatal rat cardiomyocytes stimulated with Iso $(1 \mu \mathrm{M})$ or LIF $(1 \mu \mathrm{M})$ or infected with a lentivirus containing a shRNA against Cdc42.

fibroblasts promotes JNK phosphorylation (22). Taken together with our observations, we can conclude that $\mathrm{Cdc} 42$ likely regulates JNK signaling directly through MEKK1 in cardiomyocytes, although we cannot rule out an additional effect from MEKK4 or other upstream kinases that might bind $\mathrm{Cdc} 42$.

Given that standard deletion of $C d c 42$ is embryonic lethal, the generation of LoxP-targeted $\mathrm{Cdc} 42$ mice was critical for determining its functional role in vivo. Previous analysis of these mice, or of mice with deletion of Cdc42GAP, revealed altered JNK signaling and effects on cell and organ size or cellular proliferation $(21,22)$. Cdc42 typically regulates cellular morphology, migration, and cell cycle progression by directly controlling actin and microtubule cytoskeleton formation and remodeling $(11,15)$. However, cardiomyocytes are unique in that they are structurally restrained and not typically capable of migration and cell division in adulthood. Not surprisingly, we failed to observe any alteration in myocyte number in adult hearts or hearts analyzed at 7 days after birth using the $\alpha$-MHC-cre approach, suggesting that the lack of Cdc42 in cardiomyocytes did not influence cell number. However, myocytes can change their morphology in response to stress stimulation, and Cdc42 might help regulate the hypertrophy of myocytes by controlling non-contractile actin and microtubules at the cellular periphery, where sarco- meres are added or subtracted. Indeed, $\mathrm{Cdc} 42$ can respond to signals from integrins, such as integrin $\alpha 2 / \beta 1$ in kidney cells (46). However, while we believe that loss of JNK responsiveness in $C d c^{\alpha M H C-c r e}$ mice is a primary mechanism responsible for the increased hypertrophic response, we cannot rule out other potential signaling effects downstream of $\mathrm{Cdc} 42$. Moreover, the rescue of enhanced hypertrophy in CdcaMHC-cre mice with MKK7 overexpression does not prove that JNK is a direct pathway recipient of Cdc42 signaling in controlling cardiac hypertrophy, as these effects could be parallel.

The observation that most $C d c^{\alpha M H C-c r e}$ mice died during exercise stimulation was unexpected, as was the greater hypertrophic response. Even though other indices of pathologic hypertrophy were not observed in $C d c^{\alpha M H C-c r e}$ mice after exercise, such as fibrosis and a reduction in cardiac performance, the increase in mortality suggests some sort of maladaptation (although we were not able to determine the exact defect). While physiological hypertrophy is certainly nonpathological in humans, too much of a physiological stimulus might be maladaptive (47). Indeed, overexpression of myristoylated or phosphomimetic forms of AKT1, a major transducer of physiological hypertrophy, induces pathological features in the heart such as interstitial fibrosis and impaired cardiac function $(48,49)$. In this manner, even physio- 
A

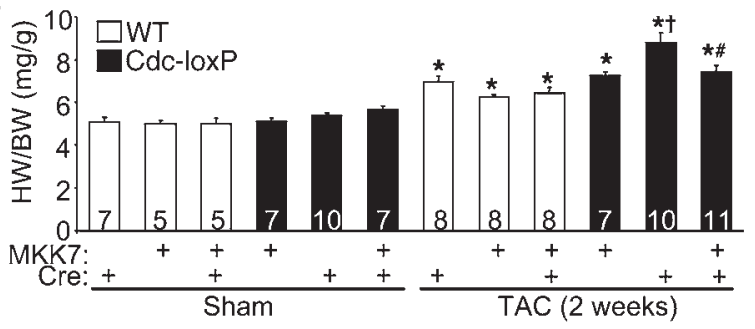

C

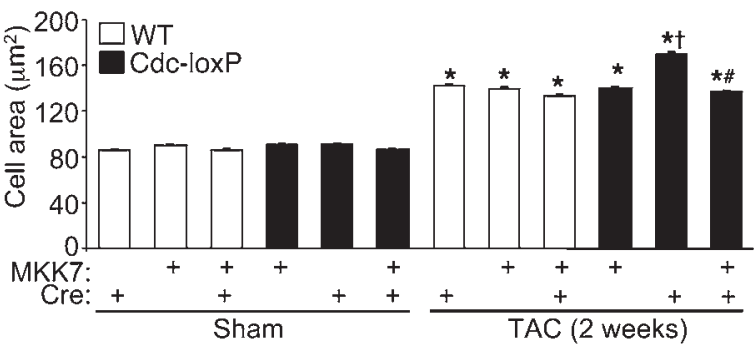

E

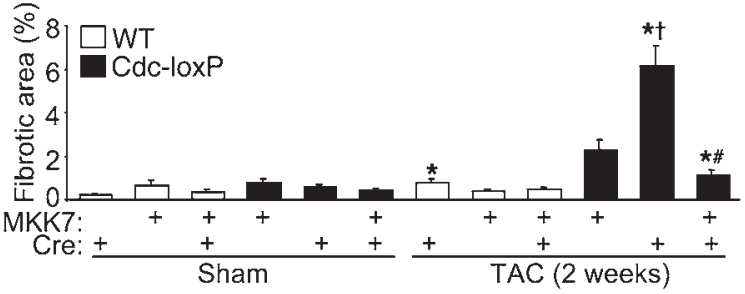

B

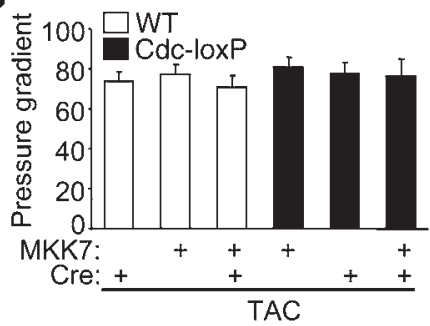

D

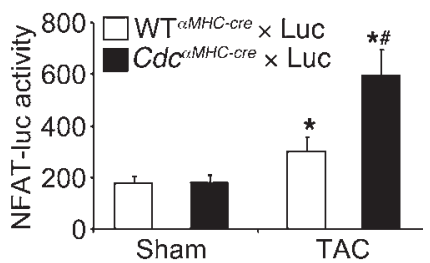

F

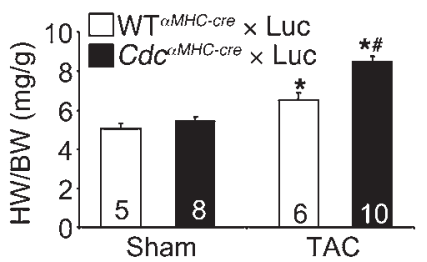

Figure 6

Restoring JNK activity in CdcaMHC-cre mice with an MKK7 transgene normalizes the cardiac hypertrophic response. (A) Quantitation of HW/BW

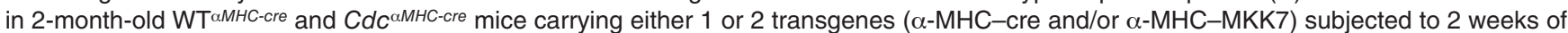
TAC or a sham procedure. The number of mice analyzed in each group is shown inside the bars. ${ }^{\star} P<0.05$ versus sham; ${ }^{\dagger} P<0.05$ versus TAC WT $\alpha$ MHC-cre; \# $P<0.05$ versus TAC Cdc ${ }^{\alpha M H C-c r e}$. (B) Quantitation of TAC pressure gradients in the different experimental groups described in A. (C) Quantitation of myocyte surface areas from histological sections of the different groups subjected to sham or TAC for 2 weeks $(n \geq 500$ myocytes from at least 3 mice in each group) ${ }^{*} P<0.05$ versus sham; ${ }^{\dagger} P<0.05$ versus TAC WT ${ }^{\alpha M H C}$-cre; $\# P<0.05$ versus TAC Cdc ${ }^{\alpha M H C}$-cre. (D) Quantitation of luciferase activity in 2-month-old WT ${ }^{\alpha M H C-c r e}$ and Cdc ${ }^{\alpha M H C-c r e}$ mice carrying the NFAT luciferase reporter transgene subjected to 2 weeks of TAC or sham. ${ }^{*} P<0.05$ versus sham; $\# P<0.05$ versus TAC WT $\alpha M H C-c r e$. (E) Quantitation of fibrosis in Masson's trichrome-stained histological heart sections from sham- and TAC-operated mice shown in $\mathbf{A}$ ( $n=3$ mice in each group). ${ }^{*}<0.05$ versus sham; ${ }^{\dagger} P<0.05$ versus TAC WT ${ }^{\alpha M H C}$-cre; $\# P<0.05$ versus TAC Cdc ${ }^{\alpha M H C-c r e}$. (F) Quantitation of HW/BW in sham-operated double-transgenic WT ${ }^{\alpha M H C-c r e}$ and Cdc ${ }^{\alpha M H C-c r e}$ mice subjected to sham operation or TAC for 2 weeks. ${ }^{*} P<0.05$ versus sham; ${ }^{\#} P<0.05$ versus TAC WT ${ }^{\alpha M H C}$-cre.

logic stimulation of hypertrophy could have a maladaptive component that is normally counteracted by protective pathways. $\mathrm{Cdc} 42$ signaling is potentially one of those protective pathways, such that rigorous exercise training without this effector leads to either arrhythmia and sudden death or greater hypertrophy. Thus, Cdc42 likely plays a unique signaling role in the heart, whereby its activation in response to both pathologic and physiologic stimuli is beneficial and antihypertrophic. This suggests that pharmacologic strategies designed to increase Cdc 42 activity might be of therapeutic value in humans.

\section{Methods}

Animal models. All experimental procedures with animals were approved by the Institutional Animal Care and Use Committee of the Cincinnati Children's Hospital Medical Center. Cdc42-LoxP-targeted mice were described previously $(17,19,20) . C d c 42^{\mathrm{WT} / \mathrm{WT}}$ and $C d c 42^{\text {loxP/loxP }}$ mice were crossed with cardiac-specific $\alpha$-MHC-cre transgenic mice (50) and labeled WT ${ }^{\alpha M H C-c r e}$ and $C d c^{\alpha M H C-c r e}$. Cardiac-specific MKK7 and NFAT luciferase reporter transgenic mice were previously described $(28,51)$. The transgenic lines
$(\mathrm{FVB} / \mathrm{N})$ were back-crossed for at least 6 generations into the C57BL/6 background, which is the background of the Cdc42-LoxP mice. $m d x$ mice (C57BL/10) were purchased from The Jackson Laboratory. The TAC surgical procedure for cardiac pressure overload stimulation was previously described (51). Cardiac ventricular performance and chamber dimensions were assayed by trans-thoracic echocardiography (50). TAC pressure gradients were determined by Doppler echocardiography and calculated as $4 \times V_{\max }{ }^{2}$, where $V_{\max }$ is the velocity of the blood across the constriction. Alzet minipumps (Durect Corp.) were used to release Ag and PE (respectively, $432 \mu \mathrm{g} / \mathrm{kg} / \mathrm{d}$ and $100 \mathrm{mg} / \mathrm{kg} / \mathrm{d}$ dissolved in $150 \mathrm{mM} \mathrm{NaCl}, 0.01 \mathrm{~N}$ acetic acid) for 2 weeks and were implanted subcutaneously following a routine surgical procedure (52). To assess exercise-induced physiological hypertrophy, mice were subjected to swimming for 3 weeks (51). The protocol consisted of a conditioning period of 8 days (10-minute-increment increases every day until two 90-minute sessions were achieved daily). EBD solution (2\%) was dissolved in sterile PBS and injected intraperitoneally (50 $\mu \mathrm{l} / 10 \mathrm{~g}$ body weight). Mice were euthanized $48 \mathrm{~h}$ after injection (53). Mice were sacrificed by $\mathrm{CO}_{2}$ asphyxiation, and organs weights were determined. $\mathrm{HW} / \mathrm{BW}$ and $\mathrm{LW} / \mathrm{BW}$ were expressed in $\mathrm{mg} / \mathrm{g}$. 
Histological analysis. Hearts were collected, fixed in 10\% formalin (PBS buffered), dehydrated, and embedded in paraffin. Global heart architecture was determined from longitudinal 5 - $\mu \mathrm{m}$ deparaffinized sections stained with H\&E. Fibrosis was detected with Masson's Trichrome staining. For cell area measurements, $5-\mu \mathrm{m}$ heart sections were deparaffinized and stained with FITC-labeled lectin from Triticum vulgaris (Sigma-Aldrich) and TO-PRO-3 iodine (Invitrogen). Surface areas were quantified across centered myocytes using Image 1.33 software (Scion Corp.). A minimum of 500 myocytes from 3 different animals was quantified for each experimental group. TUNEL apoptotic rates following 1 week of TAC and swimming exercise were determined using the TMR Red In Situ Detection Kit according to the manufacturer's instructions (Roche Diagnostics). Membranes and nuclei were counterstained with FITC-labeled lectin and TO-PRO-3 iodine (50). A minimum of 100,000 myocytes from 3 different animals were analyzed for each experimental group. Cryosections $(5 \mu \mathrm{m})$ of hearts from EBD-injected mice were counterstained with FITC-labeled lectin to visualize membranes and EBD-positive cells. A minimum of 100,000 myocytes from 3 different animals was quantified for each experimental group.

NFAT reporter assays in vivo. Hearts from NFAT-luciferase reporter transgenic mice were homogenized in lysis buffer $\left(100 \mathrm{mM} \mathrm{KPO}_{4}, 0.5 \% \mathrm{NP}-40\right.$, $1 \mathrm{mM}$ DTT), and luciferase activity was assayed in luciferase buffer $(100 \mathrm{mM}$ Tris $\mathrm{HCl} \mathrm{pH}$ 7.5, $10 \mathrm{mM} \mathrm{Mg}$ acetate, $1 \mathrm{mM}$ EDTA, $0.1 \mathrm{mM}$ luciferin, $0.3 \mathrm{mM}$ ATP). Luciferase activity was expressed as relative luciferase units per microgram of protein (51).

Fibrosis quantification. Collagen deposition in the heart stained by Masson's trichrome (blue) was quantified using Metamorph 6.1 software (Universal Imaging Corp.) as previously described (54). Blue pixels were expressed as a percentage of the entire picture surface. A total of 5 pictures covering the entire heart section were analyzed in each of at least 3 mice per experimental group (magnification, $\times 100$ ).

Cell culture and infection. Primary neonatal rat cardiomyocytes were prepared from 1- to 2-day-old Sprague-Dawley rat pups as previously described (55). Cardiomyocytes were infected for 24 hours with a human Cdc42 shRNA lentivirus that was identical in sequence to the rat (Sigma-Aldrich) in medium containing $10 \%$ serum and $8 \mathrm{mg} / \mathrm{ml}$ Polybrene (Sigma-Aldrich). Thirty-six hours later, cells were stimulated for 10 minutes in serum-free medium containing $1 \mu \mathrm{M}$ Iso, Ag, LIF, or PE and harvested for Western blotting or activity assays.

Western blotting, JNK kinase assay, and small G protein activity assays. For Western blotting, hearts were homogenized in a modified RIPA buffer (10 mM Tris- $\mathrm{HCl} \mathrm{pH}$ 7.5, $150 \mathrm{mM} \mathrm{NaCl}, 4 \%$ glycerol, $0.5 \mathrm{mM} \mathrm{Na}_{2} \mathrm{~S}_{2} \mathrm{O}_{5}$, $1 \%$ Triton $\mathrm{X}-100,0.1 \% \mathrm{Na}$ deoxycholate, $0.05 \% \mathrm{SDS}$ ) containing protease inhibitors and phosphatase inhibitor cocktails I and II (Roche Diagnostics) and centrifuged at $12,000 \mathrm{~g}$ for 15 minutes. Remaining Western blotting conditions were described previously (55). Phosphorylated and standard antibodies against ERK1/2, p38, PAK1/2, and Akt and standard antibod- ies against GSK3 $\alpha / \beta$, JNK1/2, MKK7, and MKK4 were from Cell Signaling Biotechnology. Antibodies directed against Cdc42 and phospho-FAK, were purchased from BD Biosciences. Antibodies directed against total FAK and Rac1, phospho-MKK7, and MKK4 were from Upstate Biotechnologies. RhoA, GAPDH, and phospho-MEKK1 antibodies were purchased from Santa Cruz Biotechnology Inc. MEKK1 total antibody and phosphoJNK1/2 were purchased from Invitrogen and Promega, respectively. The JNK kinase assay was performed as previously described using GST-cJun as a substrate (28). The Rac1-GTP and Cdc42-GTP pull-down assays were performed as previously described with minor modifications (56). For the Cdc42-GTP activity assay, cells were lysed as described earlier and the same buffer was used throughout the washes (55). Quantification of blots was performed with ImageQuant software (GE Healthcare).

RT-PCR. RNA $(1 \mu \mathrm{g})$ was subjected to semi-quantitative RT-PCR using SuperScript One-Step (Invitrogen) using primers for L7 (5'-GAAGCTCATCTATGAGAAGGC and 5'-AAGACGAAGGAGCTGCAGAAC), B-type natriuretic peptide (BNP) (5'-CTGCTGGAGCTGATAAGAGA and 5'-AGTCAGAACTGGAGTCTCC), $\beta$-MHC ( $5^{\prime}$-ACCTACCAGACAGAGGAAGA and $5^{\prime}$-TTGCAAAGAGTCCAGGTCTGAG), ANP (5'-GCCCTGAGTGAGCAGACTG and $5^{\prime}$-CGGAAGCTGTTGCAGCCTA), and skeletal $\alpha$-actin ( $\alpha$-Ska) (5'-AGAAGGAGATCACAGCTCTG and 5'-TACACGTCAAAAACAGGCGC). Reactions consisted of an initial RT reaction of 30 minutes at $50^{\circ} \mathrm{C}$, immediately followed by PCR of 20 or 25 cycles $\left(94^{\circ} \mathrm{C}\right.$ for $20 \mathrm{sec}-$ onds; $54^{\circ} \mathrm{C}$ for 40 seconds, and $72^{\circ} \mathrm{C}$ for 45 seconds).

Statistics. Means \pm SEM are presented. Differences between 2 experimental groups with normal distribution were analyzed by unpaired Student's $t$ test. Parametric ANOVA (coupled to Student Newman Keuls post-hoc test) was applied to normally distributed values. Differences between non-normally distributed groups were tested with Kruskal-Wallis ANOVA (Dunn's post-hoc test) using SigmaStat 3.5 software. $P<0.05$ was considered significant.

\section{Acknowledgments}

This work was supported by the NIH (grants to J.D. Molkentin and Y. Zheng). This work was also supported by an international grant in heart failure research from the Fondation Leducq (to J.D. Molkentin).

Received for publication October 8, 2008, and accepted in revised form July 8, 2009.

Address correspondence to: Jeffery D. Molkentin, Cincinnati Children's Hospital Medical Center, Molecular Cardiovascular Biology, Howard Hughes Medical Institute, 240 Albert Sabin Way, MLC 7020, Cincinnati, Ohio 45229, USA. Phone: (513) 636-3557; Fax (513) 636-5958; E-mail: jeff.molkentin@cchmc.org.
1. Claycomb, W.C. 1983. Cardiac muscle cell proliferation and cell differentiation in vivo and in vitro. Adv. Exp. Med. Biol. 161:249-265.

2. Levy, D., Garrison, R.J., Savage, D.D., Kannel, W.B., and Castelli, W.P. 1990. Prognostic implications of echocardiographically determined left ventricular mass in the Framingham Heart Study. N. Engl. J. Med. 322:1561-1566.

3. Ho, K.K., Pinsky, J.L., Kannel, W.B., and Levy, D. 1993. The epidemiology of heart failure: the Framingham Study. J. Am. Coll. Cardiol. 22:6A-13A.

4. Molkentin, J.D., et al. 1998. A calcineurin-dependent transcriptional pathway for cardiac hypertrophy. Cell. 93:215-228.

5. Heineke, J., and Molkentin, J.D. 2006. Regulation of cardiac hypertrophy by intracellular signalling pathways. Nat. Rev. Mol. Cell Biol. 7:589-600.
6. Liang, Q., and Molkentin, J.D. 2003. Redefining the roles of $\mathrm{p} 38$ and JNK signaling in cardiac hypertrophy: dichotomy between cultured myocytes and animal models. J. Mol. Cell. Cardiol. 35:1385-1394.

7. Bueno, O.F., and Molkentin, J.D. 2002. Involvement of extracellular signal-regulated kinases $1 / 2$ in cardiac hypertrophy and cell death. Circ. Res. 91:776-781.

8. Brown, J.H., Del Re, D.P., and Sussman, M.A. 2006. The Rac and Rho hall of fame: a decade of hypertrophic signaling hits. Circ. Res. 98:730-742.

9. Lezoualc'h, F., Metrich, M., Hmitou, I., Duquesnes, N., and Morel, E. 2008. Small GTP-binding proteins and their regulators in cardiac hypertrophy. J. Mol. Cell. Cardiol. 44:623-632.

10. Adams, A.E., Johnson, D.I., Longnecker, R.M., Sloat, B.F., and Pringle, J.R. 1990. CDC42 and CDC43, two additional genes involved in budding and the establishment of cell polarity in the yeast Saccharomyces cerevisiae. J. Cell Biol. 111:131-142.

11. Jaffe, A.B., and Hall, A. 2005. Rho GTPases: biochemistry and biology. Annu. Rev. Cell Dev. Biol. 21:247-269.

12. Olofsson, B. 1999. Rho guanine dissociation inhibitors: pivotal molecules in cellular signalling. Cell Signal. 11:545-554.

13. Schmidt, A., and Hall, A. 2002. Guanine nucleotide exchange factors for Rho GTPases: turning on the switch. Genes Dev. 16:1587-1609.

14. Bernards, A. 2003. GAPs galore! A survey of putative Ras superfamily GTPase activating proteins in man and Drosophila. Biochim. Biophys. Acta. 1603:47-82.

15. Etienne-Manneville, S., and Hall, A. 2002. Rho GTPases in cell biology. Nature. 420:629-635. 
16. Wu, X., et al. 2006. Cdc42 controls progenitor cell differentiation and beta-catenin turnover in skin. Genes Dev. 20:571-585.

17. Chen, L., et al. 2006. Cdc42 deficiency causes Sonic hedgehog-independent holoprosencephaly. Proc. Natl. Acad. Sci. U. S. A. 103:16520-16525.

18. Cappello, S., et al. 2006. The Rho-GTPase cdc42 regulates neural progenitor fate at the apical surface. Nat. Neurosci. 9:1099-1107.

19. Yang, L., et al. 2007. Rho GTPase Cdc42 coordinates hematopoietic stem cell quiescence and niche interaction in the bone marrow. Proc. Natl. Acad. Sci. U. S. A. 104:5091-5096

20. Yang, L., et al. 2007. Cdc42 critically regulates the balance between myelopoiesis and erythropoiesis. Blood. 110:3853-3861.

21. Wang, L., Yang, L., Debidda, M., Witte, D., and Zheng, Y. 2007. Cdc42 GTPase-activating protein deficiency promotes genomic instability and premature aging-like phenotypes. Proc. Natl. Acad. Sci. U. S. A. 104:1248-1253.

22. Yang, L., Wang, L., and Zheng, Y. 2006. Gene targeting of $\mathrm{Cdc} 42$ and Cdc42GAP affirms the critical involvement of $\mathrm{Cdc} 42$ in filopodia induction, directed migration, and proliferation in primary mouse embryonic fibroblasts. Mol. Biol. Cell. 17:4675-4685.

23. Nagai, T., et al. 2003. Cdc42 plays a critical role in assembly of sarcomere units in series of cardiac myocytes. Biochem. Biophys. Res. Commun. 305:806-810.

24. Chen, F., et al. 2000. Cdc42 is required for PIP(2)induced actin polymerization and early development but not for cell viability. Curr. Biol. 10:758-765.

25. Bishop, A.L., and Hall, A. 2000. Rho GTPases and their effector proteins. Biochem. J. 348:241-255.

26. Sadoshima, J., et al. 2002. The MEKK1-JNK pathway plays a protective role in pressure overload but does not mediate cardiac hypertrophy. J. Clin. Invest. 110:271-279.

27. Liang, Q., et al. 2003. c-Jun N-terminal kinases (JNK) antagonize cardiac growth through crosstalk with calcineurin-NFAT signaling. EMBO J. 22:5079-5089.

28. Kaiser, R.A., et al. 2005. Genetic inhibition or activation of JNK1/2 protects the myocardium from ischemia-reperfusion-induced cell death in vivo. J. Biol. Chem. 280:32602-32608.

29. Chow, C.W., Rincon, M., Cavanagh, J., Dickens, M., and Davis, R.J. 1997. Nuclear accumulation of NFAT4 opposed by the JNK signal transduction pathway. Science. 278:1638-1641.

30. Porter, C.M., Havens, M.A., and Clipstone, N.A. 2000. Identification of amino acid residues and protein kinases involved in the regulation of NFATc subcellular localization. J. Biol. Chem. 275:3543-3551

31. Zheng, M., et al. 2004. Sarcoplasmic reticulum calcium defect in Ras-induced hypertrophic cardiomyopathy heart. Am. J. Physiol. Heart Circ. Physiol. 286:H424-H433.

32. Sussman, M.A., et al. 2000. Altered focal adhesion regulation correlates with cardiomyopathy in mice expressing constitutively active rac1. J. Clin. Invest. 105:875-886

33. Satoh, M. et al. 2006. Requirement of Rac1 in the development of cardiac hypertrophy. Proc. Natl. Acad. Sci. U. S. A. 103:7432-7437.

34. Sah, V.P., et al. 1999. Cardiac-specific overexpression of RhoA results in sinus and atrioventricular nodal dysfunction and contractile failure. J. Clin. Invest. 103:1627-1634.

35. Fiedler, B., et al. 2002. Inhibition of calcineurinNFAT hypertrophy signaling by cGMP-dependent protein kinase type I in cardiac myocytes. Proc. Natl. Acad. Sci. U. S. A. 99:11363-11368.

36. Kass, D.A., Champion, H.C., and Beavo, J.A. 2007. Phosphodiesterase type 5: expanding roles in cardiovascular regulation. Circ. Res. 101:1084-1095.

37. Braz, J.C., et al. 2003. Targeted inhibition of p38 MAPK promotes hypertrophic cardiomyopathy through upregulation of calcineurin-NFAT signaling. J. Clin. Invest. 111:1475-1486.

38. Taniike, M., et al. 2008. Apoptosis signal-regulating kinase $1 / \mathrm{p} 38$ signaling pathway negatively regulates physiological hypertrophy. Circulation. 117:545-552.

39. Liao, P., et al. 2001. The in vivo role of p38 MAP kinases in cardiac remodeling and restrictive cardiomyopathy. Proc. Natl. Acad. Sci. U. S. A. 98:12283-12288.

40. Petrich, B.G., et al. 2004. Targeted activation of cJun $\mathrm{N}$-terminal kinase in vivo induces restrictive cardiomyopathy and conduction defects. J. Biol. Chem. 279:15330-15338.

41. Choukroun, G., et al. 1999. Regulation of cardiac hypertrophy in vivo by the stress-activated protein kinases/c-Jun $\mathrm{NH}(2)$-terminal kinases. J. Clin. Invest. 104:391-398.

42. Minamino, T., et al. 2002. MEKK1 is essential for cardiac hypertrophy and dysfunction induced by Gq. Proc. Natl. Acad. Sci. U. S. A. 99:3866-3871.
43. Fanger, G.R., Johnson, N.L., and Johnson, G.L. 1997. MEK kinases are regulated by EGF and selectively interact with Rac/Cdc42. EMBO J. 16:4961-4972.

44. Coso, O.A., et al. 1995. The small GTP-binding proteins Rac1 and Cdc42 regulate the activity of the JNK/SAPK signaling pathway. Cell. 81:1137-1146.

45. Minden, A., Lin, A., Claret, F.X., Abo, A., and Karin, M. 1995. Selective activation of the JNK signaling cascade and c-Jun transcriptional activity by the small GTPases Rac and Cdc42Hs. Cell. 81:1147-1157.

46. Yeh, Y.C., Wang, C.Z., and Tang, M.J. 2009. Discoidin domain receptor 1 activation suppresses alpha(2)beta(1) integrin-dependent cell spreading through inhibition of Cdc42 activity. J. Cell. Physiol. 218:146-156.

47. Shiojima, I., and Walsh, K. 2006. Regulation of cardiac growth and coronary angiogenesis by the Akt/ PKB signaling pathway. Genes Dev. 20:3347-3365.

48. Matsui, T., et al. 2002. Phenotypic spectrum caused by transgenic overexpression of activated $\mathrm{Akt}$ in the heart. J. Biol. Chem. 277:22896-22901.

49. Shioi, T., et al. 2002. Akt/protein kinase B promotes organ growth in transgenic mice. Mol. Cell. Biol. 22:2799-2809.

50. Oka, T., et al. 2006. Cardiac-specific deletion of Gata4 reveals its requirement for hypertrophy, compensation, and myocyte viability. Circ. Res. 98:837-845.

51. Wilkins, B.J., et al. 2004. Calcineurin/NFAT coupling participates in pathological, but not physiological, cardiac hypertrophy. Circ. Res. 94:110-118.

52. Wilkins, B.J., et al. 2002. Targeted disruption of NFATc3, but not NFATc4, reveals an intrinsic defect in calcineurin-mediated cardiac hypertrophic growth. Mol. Cell. Biol. 22:7603-7613.

53. Shai, S.Y., et al. 2002. Cardiac myocyte-specific excision of the beta 1 integrin gene results in myocardial fibrosis and cardiac failure. Circ. Res. 90:458-464.

54. Parsons, S.A., et al. 2004. Genetic loss of calcineurin blocks mechanical overload-induced skeletal muscle fiber type switching but not hypertrophy. J. Biol. Chem. 279:26192-26200.

55. De Windt, L.J., Lim, H.W., Haq, S., Force, T., and Molkentin, J.D. 2000. Calcineurin promotes protein kinase $\mathrm{C}$ and $\mathrm{c}$-Jun $\mathrm{NH} 2$-terminal kinase activation in the heart. Cross-talk between cardiac hypertrophic signaling pathways. J. Biol. Chem. 275:13571-13579.

56. Maillet, M., et al. 2003. Crosstalk between Rap1 and Rac regulates secretion of sAPPalpha. Nat. Cell Biol. 5:633-639. 